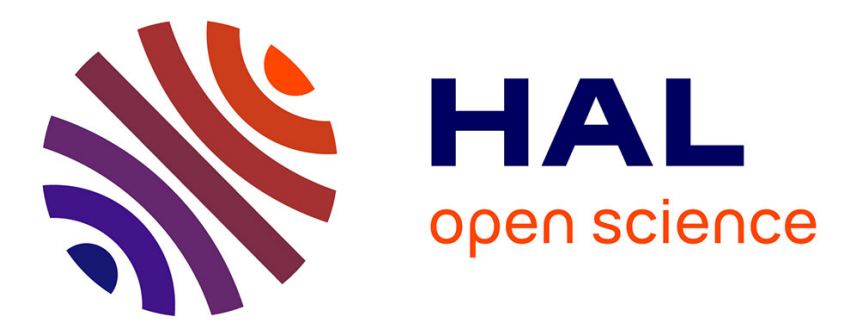

\title{
Linearization of Expectation-Based Inequality Conditions in Control for Discrete-Time Linear Systems Represented with Random Polytopes
}

\author{
Yohei Hosoe, Dimitri Peaucelle, Tomomichi Hagiwara
}

\section{To cite this version:}

Yohei Hosoe, Dimitri Peaucelle, Tomomichi Hagiwara. Linearization of Expectation-Based Inequality Conditions in Control for Discrete-Time Linear Systems Represented with Random Polytopes. Automatica, 2020, 122, pp.09228. 10.1016/j.automatica.2020.109228 . hal-03026824

\section{HAL Id: hal-03026824 \\ https://hal.science/hal-03026824}

Submitted on 26 Nov 2020

HAL is a multi-disciplinary open access archive for the deposit and dissemination of scientific research documents, whether they are published or not. The documents may come from teaching and research institutions in France or abroad, or from public or private research centers.
L'archive ouverte pluridisciplinaire HAL, est destinée au dépôt et à la diffusion de documents scientifiques de niveau recherche, publiés ou non, émanant des établissements d'enseignement et de recherche français ou étrangers, des laboratoires publics ou privés. 


\title{
Linearization of Expectation-Based Inequality Conditions in Control for Discrete-Time Linear Systems Represented with Random Polytopes *
}

\author{
Yohei Hosoe $^{\mathrm{a}, \mathrm{b}}$, Dimitri Peaucelle ${ }^{\mathrm{b}}$, Tomomichi Hagiwara ${ }^{\mathrm{a}}$ \\ ${ }^{\mathrm{a}}$ Kyoto University, Nishikyo-ku, Kyoto 615-8510, Japan \\ ${ }^{\mathrm{b}}$ LAAS-CNRS, Univ. Toulouse, CNRS, Toulouse, France
}

\begin{abstract}
A technique of linearizing what we call expectation-based inequality conditions is proposed for control of discrete-time linear systems with stochastic dynamics. In particular, the coefficient random matrices of the systems are assumed to be represented with random polytopes, and the linearization technique is discussed so as to appropriately deal with associated uncertainties. Our expectation-based inequality is an inequality that involves decision variables contained in the expectation operation, and has a unique difficulty in direct linearization, in general. Hence, two key lemmas are provided so that the decision variables can be taken out from the expectation operation. The combinational use of such lemmas and the conventional linear matrix inequality (LMI) techniques, which is nothing but our linearization technique, is expected to be useful for transforming various kinds of expectation-based nonlinear inequality into numerically solvable standard LMIs. As a demonstration, new robust stability conditions are derived with the technique, whose effectiveness is confirmed numerically.
\end{abstract}

Key words: Stochastic dynamics; discrete-time linear systems; LMI; random polytopes; robust control.

\section{Introduction}

In this paper, we deal with a class of discrete-time linear systems with stochastic dynamics described by random coefficient matrices. Such systems can be seen as a discrete-time linear case of random dynamical systems in the field of analytical dynamics (Arnold, 1998). The system class is useful for representing the influence of various stochastic phenomena such as packet interarrival times (Paxson \& Floyd, 1995) and failure occurrences (Finkelstein, 2008) in system dynamics. In addition, it is also considered to be compatible with the statistical approaches for estimation and modeling such as the ensemble Kalman filter (Evensen, 2003), whose direction of research would have a potential of being a trend toward future control technologies.

As a traditional class of systems with stochastic dynamics that have been dealt with in the field of control,

\footnotetext{
* This paper was not presented at any IFAC meeting. Corresponding author Y. Hosoe. Tel. +81-75-383-2253.

Email addresses: hosoe@kuee.kyoto-u.ac.jp (Yohei Hosoe), peaucelle@laas.fr (Dimitri Peaucelle), hagiwara@kuee.kyoto-u.ac.jp (Tomomichi Hagiwara).
}

Markov jump systems (Costa et al., 2005) are well known, whose dynamics is determined by a Markov chain. Although most of earlier studies on the systems were assuming that the underlying Markov chain only has finite modes (i.e., given with a countable state space), Costa \& Figueiredo (2014) weakened this part of assumption so that a more general stationary Markov process (i.e., that with an uncountable state space) can be dealt with, through alternatively assuming the boundedness of the supports of random coefficient matrices (depending on the process). The research team involved in the present paper, on the other hand, has studied control of systems with dynamics determined by an i.i.d. stochastic process (Hosoe et al., 2018; Hosoe \& Hagiwara, 2019), which is the time sequence of random vectors that are independent and identically distributed (i.i.d.). One might consider that our system class is included in that for Markov jump systems, since i.i.d. processes are a special case of stationary Markov processes. However, it is not true because the random coefficient matrices in our studies are allowed to have unbounded supports (e.g., normally distributed coefficients can be dealt with). Hence, the results for Markov jump systems and those in this paper do not cover each 
other, in general.

In this paper, we propose a technique useful for deriving numerically-tractable inequality conditions for control of systems with the dynamics determined by an i.i.d. process. In particular, to take account of possibilities of the presence of modeling errors even in such stochastic systems, we assume uncertainties in the random coefficient matrices that are represented with random polytopes (Hug, 2013), as in Hosoe et al. (2018). In Hosoe \& Hagiwara (2019), a Lyapunov inequality condition characterizing nominal stability was proposed and proved to be necessary and sufficient. However, the inequality involves decision variables contained in the expectation operation, which we call an expectation-based inequality. Such inequalities are difficult to transform toward advanced control compared with the conventional linear matrix inequality (LMI) conditions for deterministic systems (Boyd et al., 1994; Ebihara et al., 2015). Hence, Hosoe et al. (2018) discussed robust stability analysis and synthesis for the systems represented with random polytopes only under the approximation treatment of the distribution of the underlying i.i.d. process with its samples. The technique proposed in the present paper resolves this inconvenient situation in a direct fashion.

It is also remarked that the class of the systems with dynamics determined by an i.i.d. process is essentially the same as that of the systems with white parameters studied in De Koning (1992); Van Willigenburg \& De Koning (1999) (which is a generalization of the systems with multiplicative noise (Boyd et al., 1994)). The contributions of our study compared to those earlier results mainly lie in our (re-)developing control theory for the systems based on LMIs through some new treatment of expectations. Our direction of the study naturally enables us, e.g., to achieve multi-objective control and to deal with random polytopes for the stochastic systems, as is the case with deterministic systems. The latter advantage about ease of handling of random polytopes will be clearer through the arguments in this paper.

The contents of this paper are as follows. Section 2 briefly describes the discrete-time linear systems represented with random polytopes. Section 3 shows two key lemmas, and extends the Lyapunov inequality in Hosoe \& Hagiwara (2019) toward robust stability by using the lemmas. In particular, the obtained robust stability condition is reformulated as a standard LMI. The same technique of using the two lemmas is also applied to robustly stabilizing state feedback synthesis in Section 4. Then, Section 5 compares this new approach for robust control with our earlier approach in Hosoe et al. (2018) through a numerical example.

We use the following notation in this paper. The set of real numbers, that of positive real numbers and that of non-negative integers are denoted by $\mathbf{R}, \mathbf{R}_{+}$and $\mathbf{N}_{0}$, respectively. The set of $n$-dimensional real column vectors and that of $m \times n$ real matrices are denoted by $\mathbf{R}^{n}$ and $\mathbf{R}^{m \times n}$, respectively. The set of $n \times n$ symmetric matrices and that of $n \times n$ positive definite matrices are denoted by $\mathbf{S}^{n \times n}$ and $\mathbf{S}_{+}^{n \times n}$, respectively. The identity matrix of size $n$ is denoted by $I_{n}$; the subscript will be dropped when the size is obvious. The Euclidean norm of the vector $(\cdot)$ is denoted by $\|(\cdot)\|$. The vectorization of the matrix $(\cdot)$ in the row direction is denoted by $\operatorname{row}(\cdot)$, i.e., $\operatorname{row}(\cdot):=\left[\operatorname{row}_{1}(\cdot), \ldots, \operatorname{row}_{m}(\cdot)\right]$, where $m$ is the number of rows of the matrix and $\operatorname{row}_{i}(\cdot)$ denotes the $i$ th row. The Kronecker product is denoted by $\otimes$. The (block) diagonal matrix is denoted by $\operatorname{diag}(\cdot)$. For the real square matrix $M, \operatorname{He}(M):=M+M^{T}$, where $M^{T}$ denotes the transpose of $M$. The expectation (i.e., the expected value) of the random variable $(\cdot)$ is denoted by $E[(\cdot)]$; this notation is also used for the expectation of a random matrix. If $s$ is a random variable obeying the distribution $D$, then we represent it as $s \sim D$.

\section{Discrete-Time Linear Systems Represented with Random Polytopes}

Let us consider the $Z$-dimensional discrete-time stochastic process $\xi=\left(\xi_{k}\right)_{k \in \mathbf{N}_{0}}$ satisfying the following assumption.

Assumption $1 \xi_{k}$ is independent and identically distributed (i.i.d.) with respect to $k \in \mathbf{N}_{0}$.

This assumption naturally makes $\xi$ stationary and ergodic (Klenke, 2014). The support of $\xi_{k}$ is denoted by $\boldsymbol{\Xi}$. By definition, $\boldsymbol{\Xi} \subset \mathbf{R}^{Z}$, and $\boldsymbol{\Xi}$ corresponds to the set of values that $\xi_{k}$ can take.

With such a process $\xi$, consider the discrete-time linear system

$$
x_{k+1}=A\left(\xi_{k}\right) x_{k}
$$

where $x_{k} \in \mathbf{R}^{n}, A: \boldsymbol{\Xi} \rightarrow \mathbf{R}^{n \times n}$, and the initial state $x_{0}$ is assumed to be deterministic. This system class is general in the sense that any discrete-time linear finitedimensional system with stochastic dynamics can be described by representation (1) with $\xi$ satisfying Assumption 1 if the random coefficient matrix (denoted by $A_{k}$ ) determining the stochastic dynamics is i.i.d. with respect to the discrete time; in such a case, we can always take $A(\cdot)$ and $\xi$ such that $A\left(\xi_{k}\right)=A_{k}$.

In this paper, we assume that the random coefficient matrix $A\left(\xi_{k}\right)$ in (1) has an uncertainty and is represented by

$$
A\left(\xi_{k}\right)=A\left(\xi_{k} ; \theta\right):=\sum_{l=1}^{L} \theta^{(l)} A^{(l)}\left(\xi_{k}\right)
$$


with a deterministic uncertain parameter vector $\theta$ (= $\left.\left[\theta^{(1)}, \ldots, \theta^{(L)}\right]^{T}\right)$ satisfying

$\theta \in \mathbf{E}^{L}:=\left\{\theta \in \mathbf{R}^{L}: \theta^{(l)} \geq 0(l=1, \ldots, L), \sum_{l=1}^{L} \theta^{(l)}=1\right\}$

and the given mappings $A^{(l)}: \boldsymbol{\Xi} \rightarrow \mathbf{R}^{n \times n}(l=1, \ldots, L)$ satisfying the following assumption.

Assumption 2 For each $l=1, \ldots, L$, the squares of elements of $A^{(l)}\left(\xi_{k}\right)$ are all Lebesgue integrable, i.e.,

$$
E\left[A_{i j}^{(l)}\left(\xi_{k}\right)^{2}\right]<\infty \quad(\forall i, j=1, \ldots, n),
$$

where $A_{i j}^{(l)}\left(\xi_{k}\right)$ denotes the $(i, j)$-entry of $A^{(l)}\left(\xi_{k}\right)$.

This assumption naturally makes the squares of elements of $A\left(\xi_{k}\right)=A\left(\xi_{k} ; \theta\right)$ also Lebesgue integrable for each $\theta \in \mathbf{E}^{L}$. Although Assumption 1 is stronger than that used in the earlier study (Costa \& Figueiredo, 2014), in which $\xi$ is assumed to be stationary and Markovian, Assumption 2 is weaker than another corresponding assumption used in the same study about boundedness of (not $\boldsymbol{\Xi}$ but) the support of $A\left(\xi_{k}\right)$. This implies that Assumptions 1 and 2 would lead to such results that cannot be covered with the studies on Markov jump systems, in general. The system (1)-(3) with $\xi$ satisfying Assumptions 1 and 2 has been already dealt with in our earlier study (Hosoe et al., 2018), and we call it a discrete-time linear system represented with (or characterized by) random polytopes (Hug, 2013).

The random polytope $\left\{A\left(\xi_{k} ; \theta\right)\right\}_{\theta \in \mathbf{E}^{L}}$ is a stochastic counterpart of the usual deterministic matrix polytope and is useful for representing uncertainties in random matrices. In particular, the introduced parameter $\theta$ can be reinterpreted as uncertainties in the distributions of random matrices in some cases. For example, it is stated in Hosoe et al. (2018) that the random matrix

$$
A_{k}=\left[\begin{array}{ccc}
0.6 & 0 & a_{2 k} \\
-0.4 & a_{1 k} & 0 \\
0 & -0.4 & -a_{1 k} a_{2 k}
\end{array}\right]
$$

involving the random variables $a_{1 k}$ and $a_{2 k}$ obeying respectively the uncertain continuous uniform distribution with minimum $1.1-0.3 \delta_{1}$ and maximum $1.4-0.3 \delta_{1}\left(\delta_{1} \in\right.$ $[0,1])$ (denoted by $\left.\mathrm{U}\left(1.1-0.3 \delta_{1}, 1.4-0.3 \delta_{1}\right)\right)$ and the uncertain normal distribution with mean 0.6 and standard deviation $0.3 \delta_{2}\left(\delta_{2} \in[0,1]\right)$ (denoted by $\mathrm{N}\left(0.6,\left(0.3 \delta_{2}\right)^{2}\right)$ ) can be represented in the random polytope form without loss of generality, by associating $\theta$ with the uncertainties $\delta_{1}$ and $\delta_{2}$ under some appropriate vertices.
As the stability notion for system (1)-(3), this paper uses the following second-moment exponential stability $^{1}$ (Kozin, 1969).

Definition 1 For a fixed $\theta \in \mathbf{E}^{L}$, the system (1)-(3) (satisfying Assumptions 1 and 2) is said to be exponentially stable in the second moment if there exist $a=$ $a(\theta) \in \mathbf{R}_{+}$and $\lambda=\lambda(\theta) \in(0,1)$ such that

$$
\sqrt{E\left[\left\|x_{k}\right\|^{2}\right]} \leq a\left\|x_{0}\right\| \lambda^{k} \quad\left(\forall k \in \mathbf{N}_{0}, \forall x_{0} \in \mathbf{R}^{n}\right) .
$$

If the system is (exponentially) stable for all $\theta \in \mathbf{E}^{L}$, we say that the system is (exponentially) stable robustly with respect to $\mathbf{E}^{L}$.

\section{Expectation-Based Inequality Conditions, Their Linearization, and Application in Ro- bust Stability Problem}

This section first reviews nominal stability conditions that are represented with what we call expectationbased inequalities, and then shows and discusses two key lemmas and their roles through extending the conditions toward robust stability. The inequality condition finally obtained in this section can be solved as a standard linear matrix inequality (LMI), and hence, we call the technique for such an extension using the two key lemmas a linearization technique. Our linearization technique is expected to be useful not only for the robust stability problem but also for other robust control problems associated with random polytopes.

\subsection{First Key Lemma and Its Implication}

The following theorem giving nominal stability conditions is shown in Hosoe \& Hagiwara (2019).

Theorem 1 Suppose $\xi$ satisfies Assumption 1 and $A^{(l)}\left(\xi_{k}\right)(l=1, \ldots, L)$ satisfy Assumption 2. For each $\theta \in \mathbf{E}^{L}$, the following three conditions are equivalent.

1. The system (1)-(3) is exponentially stable in the second moment.

2. There exists $P=P(\theta) \in \mathbf{S}_{+}^{n \times n}$ such that

$$
E\left[P-A\left(\xi_{0}\right)^{T} P A\left(\xi_{0}\right)\right]>0 .
$$

3. There exist $P=P(\theta) \in \mathbf{S}_{+}^{n \times n}$ and $\lambda=\lambda(\theta) \in(0,1)$ such that

$$
E\left[\lambda^{2} P-A\left(\xi_{0}\right)^{T} P A\left(\xi_{0}\right)\right] \geq 0 .
$$

\footnotetext{
1 Note that second-moment exponential stability (also called exponential mean square stability) is shown in Hosoe \& Hagiwara (2019) to be equivalent to second-moment asymptotic stability (i.e., asymptotic mean square stability) and quadratic stability for the present class of systems.
} 
The expectation-based inequality (7) is a stochastic counterpart of the Lyapunov inequality for discrete-time deterministic linear time-invariant systems. In addition, (8) is also useful when the convergence rate with respect to the sequence $\left(\sqrt{E\left[\left\|x_{k}\right\|^{2}\right]}\right)_{k \in \mathbf{N}_{0}}$ is required, since $\lambda$ in (8) corresponds to that in (6). For this reason, we will derive a robust stability condition from (8) with a technique using two lemmas shown later. However, the same technique can be used also for (7), which leads to another slightly different robust stability condition without $\lambda$.

The following is the first key lemma used in our linearization technique.

Lemma 1 For a given random vector $\xi_{0}$ (with support $\boldsymbol{\Xi})$ and a given mapping $R: \boldsymbol{\Xi} \rightarrow \mathbf{S}^{n_{0} \times n_{0}}$, the following two conditions are equivalent.

1. The following inequality holds.

$$
E\left[R\left(\xi_{0}\right)\right] \geq 0
$$

2. There exists $T: \boldsymbol{\Xi} \rightarrow \mathbf{S}^{n_{0} \times n_{0}}$ such that

$$
\begin{aligned}
& E\left[T\left(\xi_{0}\right)\right] \leq 0, \\
& T\left(\xi_{\star}\right)+R\left(\xi_{\star}\right) \geq 0 \quad\left(\forall \xi_{\star} \in \boldsymbol{\Xi}\right) .
\end{aligned}
$$

PROOF. $1 \Rightarrow 2: T(\cdot)=-R(\cdot)$ readily leads to $(10)$ and (11).

$2 \Rightarrow 1$ : It follows from (11) that for each sample of $\xi_{0}$, we have $T\left(\xi_{0}\right)+R\left(\xi_{0}\right) \geq 0$. This implies $E\left[R\left(\xi_{0}\right)\right] \geq$ $-E\left[T\left(\xi_{0}\right)\right]$ for random $\xi_{0}$, which together with (10) leads to $(9)$.

The newly introduced mapping $T$ in this lemma enables us to make the random matrix $R\left(\xi_{0}\right)$ uncontained in the expectation operation as in (10) and (11); the converse assertion (i.e., the assertion from condition 2 to condition 1) is also important and will be used soon. Although this lemma itself might seem almost trivial, it is actually very powerful in deriving inequality conditions associated with the use of random polytopes in system representation. For example, we can easily obtain the following corollary, which gives a stochastic counterpart of the LMI condition with $S$-variables (i.e., auxiliary variables) (Ebihara et al., 2015) developed for deterministic systems.

Corollary 1 For given $\xi_{0}$ (with $\boldsymbol{\Xi}$ ), $P \in \mathbf{S}_{+}^{n \times n}, N_{1}$ : $\boldsymbol{\Xi} \rightarrow \mathbf{R}^{n_{1} \times n_{0}}\left(n_{1} \geq n_{0}\right), U: \mathbf{R} \times \mathbf{S}^{n \times n} \rightarrow \mathbf{S}^{n_{1} \times n_{1}}$ and $\lambda \in(0,1)$,

$$
E\left[N_{1}\left(\xi_{0}\right)^{T} U(\lambda, P) N_{1}\left(\xi_{0}\right)\right] \geq 0
$$

holds if there exist $S \in \mathbf{R}^{n_{1} \times n_{2}}, T: \boldsymbol{\Xi} \rightarrow \mathbf{S}^{n_{0} \times n_{0}}, J:$ $\mathbf{S}^{n_{0} \times n_{0}} \rightarrow \mathbf{S}^{n_{1} \times n_{1}}$ and $N_{2}: \boldsymbol{\Xi} \rightarrow \mathbf{R}^{n_{2} \times n_{1}}$ satisfying (10) and

$$
\begin{aligned}
& J\left(T\left(\xi_{\star}\right)\right)+U(\lambda, P)+\operatorname{He}\left(S N_{2}\left(\xi_{\star}\right)\right) \geq 0 \quad\left(\forall \xi_{\star} \in \boldsymbol{\Xi}\right), \\
& N_{1}\left(\xi_{\star}\right)^{T} J(\Pi) N_{1}\left(\xi_{\star}\right)=\Pi \quad\left(\forall \Pi \in \mathbf{S}^{n_{0} \times n_{0}}, \forall \xi_{\star} \in \boldsymbol{\Xi}\right), \\
& N_{2}\left(\xi_{\star}\right) N_{1}\left(\xi_{\star}\right)=0 \quad\left(\forall \xi_{\star} \in \boldsymbol{\Xi}\right) .
\end{aligned}
$$

PROOF. By post- and pre-multiplying $N_{1}\left(\xi_{\star}\right)$ and its transpose on (13), we obtain

$$
T\left(\xi_{\star}\right)+N_{1}\left(\xi_{\star}\right)^{T} U(\lambda, P) N_{1}\left(\xi_{\star}\right) \geq 0 \quad\left(\forall \xi_{\star} \in \boldsymbol{\Xi}\right)
$$

by (14) and (15). This, together with Lemma 1, completes the proof.

The significance of this corollary lies in the linearity of the inequality condition (10) and (13) in the operator $N_{2}(\cdot)$, despite the nonlinearity of (12) in $N_{1}(\cdot)$ (which has a close connection with $N_{2}(\cdot)$ through (15)). This significance can be demonstrated by showing the following theorem, which gives a robust stability condition for the systems represented with random polytopes.

Theorem 2 Suppose $\xi$ satisfies Assumption 1 and $A^{(l)}\left(\xi_{k}\right)(l=1, \ldots, L)$ satisfy Assumption 2. The system (1)-(3) is exponentially stable in the second moment robustly with respect to $\mathbf{E}^{L}$ if there exist $S \in \mathbf{R}^{2 n \times n}$, $P^{(l)} \in \mathbf{S}_{+}^{n \times n}, T^{(l)}: \boldsymbol{\Xi} \rightarrow \mathbf{S}^{n \times n}(l=1, \ldots, L)$ and $\lambda \in(0,1)$ satisfying

$$
\begin{aligned}
& E\left[T^{(l)}\left(\xi_{0}\right)\right] \leq 0 \quad(l=1, \ldots, L), \\
& {\left[\begin{array}{cc}
T^{(l)}\left(\xi_{\star}\right)+\lambda^{2} P^{(l)} & 0 \\
0 & -P^{(l)}
\end{array}\right]+\operatorname{He}\left(S\left[A^{(l)}\left(\xi_{\star}\right) I\right]\right) \geq 0} \\
& \left(l=1, \ldots, L ; \forall \xi_{\star} \in \boldsymbol{\Xi}\right) .
\end{aligned}
$$

PROOF. Fix $\theta \in \mathbf{E}^{L}$. Fix also $l$ and take

$$
U(\lambda, \cdot)=\left[\begin{array}{cc}
\lambda^{2}(\cdot) & 0 \\
0 & -(\cdot)
\end{array}\right], N_{1}^{(l)}(\cdot)=\left[\begin{array}{c}
I \\
-A^{(l)}(\cdot)
\end{array}\right]
$$

and then

$$
J(\cdot)=\left[\begin{array}{cc}
(\cdot) & 0 \\
0 & 0
\end{array}\right], N_{2}^{(l)}(\cdot)=\left[A^{(l)}(\cdot) I\right]
$$

so that (14) and (15) with $N_{i}(\cdot)$ replaced by $N_{i}^{(l)}(\cdot)(i=$ $1,2)$ hold. Then, it follows from (18) that

$$
J\left(T^{(l)}\left(\xi_{\star}\right)\right)+U\left(\lambda, P^{(l)}\right)+\operatorname{He}\left(S N_{2}^{(l)}\left(\xi_{\star}\right)\right) \geq 0
$$




$$
\left(\forall \xi_{\star} \in \boldsymbol{\Xi}\right)
$$

Since this is true for each $l$, multiplying $\theta^{(l)}$ on this inequality and (17) and taking the sums leads to (10) and (13) with $T(\cdot)=T(\cdot ; \theta):=\sum_{i=1}^{L} \theta^{(l)} T^{(l)}(\cdot), P=$ $P(\theta):=\sum_{i=1}^{L} \theta^{(l)} P^{(l)}$ and $N_{2}(\cdot)=[A(\cdot) I]$. Furthermore, taking $N_{1}(\cdot)=\left[I-A(\cdot)^{T}\right]^{T}$ leads to (14) and (15). Hence, it follows from Corollary 1 that

$$
E\left[\left[\begin{array}{c}
I \\
-A\left(\xi_{0}\right)
\end{array}\right]^{T}\left[\begin{array}{cc}
\lambda^{2} P & 0 \\
0 & -P
\end{array}\right]\left[\begin{array}{c}
I \\
-A\left(\xi_{0}\right)
\end{array}\right]\right] \geq 0,
$$

which is nothing but (8). This together with Theorem 1 completes the proof.

Although the direct extension of the inequality condition (8) toward robust stability is difficult because of its nonlinearity in $A\left(\xi_{0}\right)$, Corollary 1 about the introduction of $S$-variables enables us to circumvent this issue as in the above proof even for the present stochastic systems case. Obviously, Lemma 1 has played a crucial role in showing Corollary 1, and hence, the above arguments indeed demonstrate an aspect of the powerfulness of Lemma 1; the lemma (and the associated corollary) is useful for deriving a sufficient inequality condition described with the vertices of the random polytope, as in the present robust stability problem.

If we restrict $S$ in (18) to be $\left[0, G^{T}\right]^{T}$ with $G \in \mathbf{R}^{n \times n}$, present Theorem 2 immediately reduces to Theorem 2 in our earlier study (Hosoe et al., 2018), which was derived with a completely different approach (i.e., without using Lemma 1). Hence, present Theorem 2 corresponds to a generalization of the earlier result and would enable us to achieve less conservative robust stability analysis. The simultaneous inequality condition consisting of expectation-based and infinitely-many matrix inequalities such as (17) and (18), however, is generally difficult to directly use for numerical analysis (even when the mappings $T^{(l)}$ are confined to some tractable class). Indeed, this was an obstacle and an analysis method using samples of $\xi_{0}$ was additionally discussed in Hosoe et al. (2018), in which it was required to evaluate the confidence level of the analysis result since the result inevitably depends on the used samples. Hence, if we stop our arguments here, the analysis based on present Theorem 2 would also have the same issue. To directly resolve this inconvenient situation so that the theoretically equivalent level of analysis (i.e., that using Theorem 2) can be performed without such a sample-based method, we provide in the next subsection the second key lemma and discuss a way of further transforming expectationbased inequality conditions. Such arguments will simultaneously lead us to a numerically-tractable inequality condition for robust stability that can be solved without the sample-based method despite no increase of the conservativeness in the corresponding robust stability analysis (compared with that using present Theorem 2).

\subsection{Second Key Lemma and Numerically-Tractable Ro- bust Stability Condition}

The following is the second key lemma in our linearization technique.

\section{Lemma 2 For matrices}

$$
\begin{aligned}
& M_{1}=\left[\begin{array}{c}
M_{11} \\
\vdots \\
M_{1 p}
\end{array}\right] \quad\left(M_{1 i} \in \mathbf{R}^{r \times m_{1}}\right), \\
& M_{2}=\left[\begin{array}{c}
M_{21} \\
\vdots \\
M_{2 q}
\end{array}\right] \quad\left(M_{2 i} \in \mathbf{R}^{r \times m_{2}}\right)
\end{aligned}
$$

and $H \in \mathbf{R}^{p \times q}$,

$$
M_{1}^{T}\left(H \otimes I_{r}\right) M_{2}=\sum_{i=1}^{p} \sum_{j=1}^{q} h_{i j} M_{1 i}^{T} M_{2 j}
$$

holds, where $h_{i j}$ is the $(i, j)$-entry of $H$.

This lemma can be shown with the direct calculation of the matrices, and corresponds to a generalization of the idea implicitly used in Hosoe \& Hagiwara (2019) for solving the expectation-based inequality conditions (7) and (8) (involving the decision variable $P$ contained in the expectation operation) as standard matrix inequalities.

Before proceeding to the description of our linearization technique for expectation-based inequalities, we demonstrate how to use Lemmas 1 and 2 through deriving a numerically-tractable LMI condition for robust stability with the lemmas. Consider the (additional) constraint ${ }^{2}$

$$
S_{2}+S_{2}^{T}-P^{(l)}>0(l=1, \ldots, L)
$$

on $P^{(l)}$ and $S=:\left[S_{1}^{T}, S_{2}^{T}\right]^{T}\left(S_{1}, S_{2} \in \mathbf{R}^{n \times n}\right)$. Then, under this constraint, the Schur complement technique (Boyd et al., 1994) ensures that the inequality in (18) is equivalent to

$T^{(l)}\left(\xi_{\star}\right)+\lambda^{2} P^{(l)}+\operatorname{He}\left(S_{1} A^{(l)}\left(\xi_{\star}\right)\right)-\left(S_{1}^{T}+S_{2} A^{(l)}\left(\xi_{\star}\right)\right)^{T}$

\footnotetext{
2 This does not actually increase the conservativeness in the associated analysis because of the positive definiteness of $P^{(l)}$ although the details are omitted.
} 


$$
\cdot\left(S_{2}+S_{2}^{T}-P^{(l)}\right)^{-1}\left(S_{1}^{T}+S_{2} A^{(l)}\left(\xi_{\star}\right)\right) \geq 0 .
$$

Hence, Lemma 1 leads us to the following corollary.

Corollary 2 For given $S=\left[S_{1}^{T}, S_{2}^{T}\right]^{T}\left(S_{1}, S_{2} \in\right.$ $\left.\mathbf{R}^{n \times n}\right), P^{(l)} \in \mathbf{S}_{+}^{n \times n}(l=1, \ldots, L)$ and $\lambda \in(0,1)$, the following two conditions are equivalent.

1. There exist $T^{(l)}: \boldsymbol{\Xi} \rightarrow \mathbf{S}^{n \times n}(l=1, \ldots, L)$ such that (17), (18) and (26) hold.

2. The following inequality and (26) hold.

$$
\begin{aligned}
& E\left[\lambda^{2} P^{(l)}+\operatorname{He}\left(S_{1} A^{(l)}\left(\xi_{0}\right)\right)-\left(S_{1}^{T}+S_{2} A^{(l)}\left(\xi_{0}\right)\right)^{T}\right. \\
& \left.\cdot\left(S_{2}+S_{2}^{T}-P^{(l)}\right)^{-1}\left(S_{1}^{T}+S_{2} A^{(l)}\left(\xi_{0}\right)\right)\right] \geq 0 \\
& (l=1, \ldots, L)
\end{aligned}
$$

This kind of transformation of inequalities using Lemma 1 has never been considered in our earlier studies and will be a key in using Lemma 2 next.

We see that each term, after appropriate expansion, in the expectation operation in $(28)$ can be represented in the form $M_{1}\left(\xi_{0}\right)^{T} H M_{2}\left(\xi_{0}\right)$ for $M_{i}\left(\xi_{0}\right)=A^{(l)}\left(\xi_{0}\right)$ or $I_{n}$. For example, $S_{1}\left(S_{2}+S_{2}^{T}-P^{(l)}\right)^{-1} S_{2} A^{(l)}\left(\xi_{0}\right)$ can be represented as in that form with $M_{1}\left(\xi_{0}\right)=I_{n}, M_{2}\left(\xi_{0}\right)=$ $A^{(l)}\left(\xi_{0}\right)$ and $H=S_{1}\left(S_{2}+S_{2}^{T}-P^{(l)}\right)^{-1} S_{2}$. Let us temporarily focus on this form of expectations. Then, by using Lemma 2 under $r=1$, this expectation can be transformed as

$$
\begin{aligned}
& E\left[M_{1}\left(\xi_{0}\right)^{T} H M_{2}\left(\xi_{0}\right)\right] \\
= & \sum_{i=1}^{n} \sum_{j=1}^{n} h_{i j} E\left[\operatorname{row}_{i}\left(M_{1}\left(\xi_{0}\right)\right)^{T} \operatorname{row}_{j}\left(M_{2}\left(\xi_{0}\right)\right)\right],
\end{aligned}
$$

where $h_{i j}$ is the $(i, j)$-entry of $H\left(\in \mathbf{R}^{n \times n}\right)$. The expectation operation in the right-hand side of this equation does not contain decision variables. Here, if we can take $\bar{X}_{A i}^{(l)} \in \mathbf{R}^{\bar{n}_{l} \times n}$ and $\bar{X}_{I i}^{(l)} \in \mathbf{R}^{\bar{n}_{l} \times n}$ (for some $\bar{n}_{l}$ ) satisfying

$$
\begin{aligned}
& E\left[\operatorname{row}_{i}\left(A^{(l)}\left(\xi_{0}\right)\right)^{T} \operatorname{row}_{j}\left(A^{(l)}\left(\xi_{0}\right)\right)\right]=\bar{X}_{A i}^{(l) T} \bar{X}_{A j}^{(l)}, \\
& E\left[\operatorname{row}_{i}\left(I_{n}\right)^{T} \operatorname{row}_{j}\left(A^{(l)}\left(\xi_{0}\right)\right)\right]=\bar{X}_{I i}^{(l) T} \bar{X}_{A j}^{(l)}, \\
& E\left[\operatorname{row}_{i}\left(I_{n}\right)^{T} \operatorname{row}_{j}\left(I_{n}\right)\right]=\bar{X}_{I i}^{(l) T} \bar{X}_{I j}^{(l)}
\end{aligned}
$$

for each $l=1, \ldots, L$, then Lemma 2 applied to the righthand side of (29) under $r=\bar{n}_{l}$ leads us to

$$
\begin{aligned}
& E\left[A^{(l)}\left(\xi_{0}\right)^{T} H A^{(l)}\left(\xi_{0}\right)\right]=\widetilde{X}_{A}^{(l) T}\left(H \otimes I_{\bar{n}_{l}}\right) \widetilde{X}_{A}^{(l)}, \\
& E\left[H A^{(l)}\left(\xi_{0}\right)\right]=\widetilde{X}_{I}^{(l) T}\left(H \otimes I_{\bar{n}_{l}}\right) \widetilde{X}_{A}^{(l)}, \\
& (H=) E[H]=\widetilde{X}_{I}^{(l) T}\left(H \otimes I_{\bar{n}_{l}}\right) \widetilde{X}_{I}^{(l)},
\end{aligned}
$$

where

$$
\tilde{X}_{A}^{(l)}=\left[\begin{array}{c}
\bar{X}_{A 1}^{(l)} \\
\vdots \\
\bar{X}_{A n}^{(l)}
\end{array}\right] \in \mathbf{R}^{n \bar{n}_{l} \times n}, \quad \tilde{X}_{I}^{(l)}=\left[\begin{array}{c}
\bar{X}_{I 1}^{(l)} \\
\vdots \\
\bar{X}_{I n}^{(l)}
\end{array}\right] \in \mathbf{R}^{n \bar{n}_{l} \times n}
$$

The right-hand sides of (33)-(35) are tractable from the viewpoint of using LMI techniques. The matrices $\bar{X}_{A i}^{(l)}$ and $\bar{X}_{I i}^{(l)}$ making this transformation possible can be obtained as follows. Take full row rank matrices $\bar{X}_{1 A}^{(l)} \in$ $\mathbf{R}^{\bar{n}_{l} \times\left(n^{2}+1\right)}(l=1, \ldots, L)$ satisfying

$$
\begin{aligned}
\bar{X}_{1 A}^{(l) T} \bar{X}_{1 A}^{(l)} & =E\left[X_{1 A}^{2(l)}\left(\xi_{0}\right)\right], \\
X_{1 A}^{2(l)}\left(\xi_{0}\right) & :=\left[1, \operatorname{row}\left(A^{(l)}\left(\xi_{0}\right)\right)\right]^{T}\left[1, \operatorname{row}\left(A^{(l)}\left(\xi_{0}\right)\right)\right] .
\end{aligned}
$$

Then, $\bar{X}_{A i}^{(l)}$ can be obtained as

$$
\begin{aligned}
& \bar{X}_{1 A}^{(l)}=:\left[\bar{X}_{1}^{(l)}, \bar{X}_{A 1}^{(l)}, \ldots, \bar{X}_{A n}^{(l)}\right] \\
& \left(\bar{X}_{1}^{(l)} \in \mathbf{R}^{\bar{n}_{l} \times 1}, \bar{X}_{A i}^{(l)} \in \mathbf{R}^{\bar{n}_{l} \times n}(i=1, \ldots, n)\right),
\end{aligned}
$$

while $\bar{X}_{I i}^{(l)}$ can be constructed as

$$
\bar{X}_{I i}^{(l)}=\bar{X}_{1}^{(l)} \operatorname{row}_{i}\left(I_{n}\right) \in \mathbf{R}^{\bar{n}_{l} \times n}(i=1, \ldots, n),
$$

which can be confirmed by a direct calculation to satisfy (30)-(32).

With the transformation of expectations (33)-(35), the inequality condition (28) can be equivalently rewritten as

$$
\begin{aligned}
& \lambda^{2} P^{(l)}+\operatorname{He}\left(\widetilde{X}_{I}^{(l) T}\left(S_{1} \otimes I_{\bar{n}_{l}}\right) \widetilde{X}_{A}^{(l)}\right) \\
& -\left\{\left(S_{1}^{T} \otimes I_{\bar{n}_{l}}\right) \widetilde{X}_{I}^{(l)}+\left(S_{2} \otimes I_{\bar{n}_{l}}\right) \widetilde{X}_{A}^{(l)}\right\}^{T} \\
& \cdot\left\{\left(S_{2}+S_{2}^{T}-P^{(l)}\right) \otimes I_{\bar{n}_{l}}\right\}^{-1} \\
& \cdot\left\{\left(S_{1}^{T} \otimes I_{\bar{n}_{l}}\right) \widetilde{X}_{I}^{(l)}+\left(S_{2} \otimes I_{\bar{n}_{l}}\right) \widetilde{X}_{A}^{(l)}\right\} \geq 0 \\
& (l=1, \ldots, L) .
\end{aligned}
$$

Since $\left(S_{2}+S_{2}^{T}-P^{(l)}\right) \otimes I_{\bar{n}_{l}}>0$, the Schur complement technique again ensures that (41) (i.e., (28)) is equivalent to

$$
\begin{aligned}
& {\left[\begin{array}{cc}
\lambda^{2} P^{(l)} & 0 \\
0 & -P^{(l)} \otimes I_{\bar{n}_{l}}
\end{array}\right]} \\
& +\operatorname{He}\left(\left[\begin{array}{c}
S_{1} \otimes \bar{X}_{1}^{(l) T} \\
S_{2} \otimes I_{\bar{n}_{l}}
\end{array}\right]\left[\begin{array}{ll}
\widetilde{X}_{A}^{(l)} & I_{n \bar{n}_{l}}
\end{array}\right]\right) \geq 0
\end{aligned}
$$




$$
(l=1, \ldots, L),
$$

where we used $\widetilde{X}_{I}^{(l)}=I_{n} \otimes \bar{X}_{1}^{(l)}$, which can be confirmed from (40) and (36). Hence, this, together with Theorem 2 and Corollary 2, leads us to the following theorem.

Theorem 3 Suppose $\xi$ satisfies Assumption 1 and $A^{(l)}\left(\xi_{k}\right)(l=1, \ldots, L)$ satisfy Assumption 2. The system (1)-(3) is exponentially stable in the second moment robustly with respect to $\mathbf{E}^{L}$ if there exist $S_{i} \in \mathbf{R}^{n \times n}(i=1,2), P^{(l)} \in \mathbf{S}_{+}^{n \times n}(l=1, \ldots, L)$ and $\lambda \in(0,1)$ satisfying (26) and (42), where $\bar{X}_{1}^{(l)}$ and $\widetilde{X}_{A}^{(l)}$ are given as (39) and (36) with full row rank matrices $\bar{X}_{1 A}^{(l)} \in \mathbf{R}^{\bar{n}_{l} \times\left(n^{2}+1\right)}$ satisfying (37) and (38).

Once $\bar{X}_{1}^{(l)}$ and $\widetilde{X}_{A}^{(l)}$ are calculated with the vertices $A^{(l)}\left(\xi_{0}\right)(l=1, \ldots, L)$, the inequality condition (26) and (42) can be seen as a standard LMI (for each fixed $\lambda)$ with the decision variables $S_{i}(i=1,2)$ and $P^{(l)}(l=1, \ldots, L)$. Hence, we can analyze robust stability by minimizing $\lambda$ with respect to the inequality condition through the bisection with respect to $\lambda$. This inequality condition is numerically more tractable than that in Theorem 2 in the sense that the sample-based method and the associated evaluation of the confidence level are not required. That is, the obtained $\lambda$ readily corresponds to an upper bound of the convergence rate with respect to $\left(\sqrt{E\left[\left\|x_{k}\right\|^{2}\right]}\right)_{k \in \mathbf{N}_{0}}$ for each $\theta \in \mathbf{E}^{L}$.

Remark 1 When $L=1, S_{1}=0$ and $S_{2}=P\left(=P^{(1)}\right)$, the inequality condition (42) essentially reduces to that for nominal stability derived in our earlier study (Hosoe \& Hagiwara, 2019). A transformation similar to that from (28) to (41) was also discussed in the study. The main differences from the earlier result in terms of this part are that the core idea of the technique used in the transformation was summarized explicitly as Lemma 2 so that the applicability of the technique is improved, and that we newly provided a key idea for dealing with the left-hand sides of (34) and (35) as well as (33) through the matrices $\widetilde{X}_{A}^{(l)}$ and $\widetilde{X}_{I}^{(l)}$. This is because the present paper dealing with robust stability came to handling the expectations of the entries of the matrix $A\left(\xi_{0}\right)$ as in (34) through the introduction of the $S$-variable, unlike the earlier study in which it sufficed to deal with the expectations of the products of these entries as in (33); for concisely dealing with these two types of expectations, introducing the matrices $\widetilde{X}_{A}^{(l)}$ and $\widetilde{X}_{I}^{(l)}$ is convenient, and is actually quite essential in the robust stabilization synthesis studied in the following section. Furthermore, the advantage of taking the full rank decomposition in (37) in reducing the associated computation cost was not claimed in the earlier study.

\subsection{Summary of Linearization Technique Using Two Key Lemmas}

In the preceding subsections, a numerically-tractable robust stability condition was derived by using Lemmas 1 and 2, which condition itself is new and useful. However, not such a specific condition but the technique used in its derivation is more significant and indeed constitutes a main contribution in this paper. The roles of the two lemmas used in the derivation can be summarized as follows.

Role of Lemma 1: To obtain $\theta$-independent (i.e., uncertainty-free) inequalities associated with the vertices of the random polytope such as (28) from $\theta$ dependent inequalities such as (8).

Role of Lemma 2: To obtain inequalities with decision variables uncontained in the expectation operation such as (42) from those with decision variables contained in the expectation operation such as (28).

Hence, even when we have only a $\theta$-dependent inequality condition with decision variables contained in the expectation operation (i.e., a $\theta$-dependent expectationbased inequality), these lemmas could lead us to a $\theta$ independent inequality condition with decision variables uncontained in the expectation operation from that original inequality condition. This is helpful for further using the conventional LMI techniques, and indeed, the LMI condition (26) and (42) was finally derived in the present robust stability problem case through the combination of the lemmas with the $S$-variable approach and the Schur complement technique. The whole technique of deriving LMI conditions in such a way with the two key lemmas is nothing but what we propose in this paper. Note that this technique itself is not specific to the present robust stability problem, and many other robust control problems could also be tackled in a similar fashion.

Remark 2 To the best of the authors' knowledge, no similar arguments arise in the field of Markov jump linear systems. This is because the inequality conditions such as the Lyapunov inequalities for standard Markov jump linear systems can always be written directly as standard matrix inequalities since any underlying expectation can be described merely as a weighted summation of a finite number of matrix terms. Hence, we encounter no obstacles in applying the standard LMI techniques such as the Schur complement technique directly to the matrix inequalities. Indeed, robust stability problems have already been discussed in de Souza (2006) for these systems only through the standard treatment. 


\section{Robustly Stabilizing State Feedback Synthe-} sis

The Linearization technique discussed in the preceding section can be easily applied to the robustly stabilizing state feedback synthesis problem for the systems represented with random polytopes. Let us consider the $Z$ dimensional process $\xi$ satisfying Assumption 1 and the associated system

$$
\begin{aligned}
& x_{k+1}=A_{\mathrm{op}}\left(\xi_{k}\right) x_{k}+B_{\mathrm{op}}\left(\xi_{k}\right) u_{k}, \\
& A_{\mathrm{op}}\left(\xi_{k}\right)=A_{\mathrm{op}}\left(\xi_{k} ; \theta\right):=\sum_{l=1}^{L} \theta^{(l)} A_{\mathrm{op}}^{(l)}\left(\xi_{k}\right), \\
& B_{\mathrm{op}}\left(\xi_{k}\right)=B_{\mathrm{op}}\left(\xi_{k} ; \theta\right):=\sum_{l=1}^{L} \theta^{(l)} B_{\mathrm{op}}^{(l)}\left(\xi_{k}\right)
\end{aligned}
$$

and (3), where $x_{k} \in \mathbf{R}^{n}, u_{k} \in \mathbf{R}^{m}, A_{\mathrm{op}}^{(l)}: \boldsymbol{\Xi} \rightarrow \mathbf{R}^{n \times n}$ and $B_{\mathrm{op}}^{(l)}: \boldsymbol{\Xi} \rightarrow \mathbf{R}^{n \times m}(l=1, \ldots, L)$. On the vertices of the coefficient matrices of the above system, we make the following assumption similar to Assumption 2.

Assumption 3 For each $l=1, \ldots, L$, the squares of elements of $A_{\mathrm{op}}^{(l)}\left(\xi_{k}\right)$ and $B_{\mathrm{op}}^{(l)}\left(\xi_{k}\right)$ are all Lebesgue integrable.

Let us also consider the state feedback

$$
u_{k}=F x_{k}
$$

with the static time-invariant gain $F \in \mathbf{R}^{m \times n}$. Then, the closed-loop system can be described by (1)-(3) with

$$
A^{(l)}\left(\xi_{k}\right)=A_{\mathrm{op}}^{(l)}\left(\xi_{k}\right)+B_{\mathrm{op}}^{(l)}\left(\xi_{k}\right) F \quad(l=1, \ldots, L) .
$$

Note that if $A_{\mathrm{op}}^{(l)}\left(\xi_{k}\right)$ and $B_{\mathrm{op}}^{(l)}\left(\xi_{k}\right)$ satisfy Assumption 3 then the above $A^{(l)}\left(\xi_{k}\right)$ also satisfy Assumption 2 (for each fixed $F$ ). This section shows an inequality condition for designing $F$ such that the closed-loop system is exponentially stable in the second moment robustly with respect to $\theta \in \mathbf{E}^{L}$.

By Theorem 2, we see that the inequality condition for robust closed-loop stability can be given by (17) and (18) with $A^{(l)}\left(\xi_{k}\right)$ in (47). Since this inequality condition is difficult to linearize for the synthesis of $F$ under the general class of $S$ even in the case with deterministic systems, we restrict the class of $S$-variables to the following form ${ }^{3}$ with a given matrix $A_{\mathrm{s}} \in \mathbf{R}^{n \times n}\left(A_{\mathrm{s}}\right.$ can actually be confined to a Schur stable matrix without

\footnotetext{
3 This form itself does not actually decrease generality of $S$ if $A_{\mathrm{s}}$ is also regarded as a decision variable.
}

loss of generality, although the details are omitted).

$$
S=\operatorname{diag}(G, G)\left[\begin{array}{c}
A_{\mathrm{s}}^{T} \\
I_{n}
\end{array}\right] \quad\left(G \in \mathbf{R}^{n \times n}\right)
$$

Then, $G$ is nonsingular from $P^{(l)}>0$ and the lower right block of (18), and applying the congruence transformations using $G^{-1}$ and $\operatorname{diag}\left(G^{-1}, G^{-1}\right)$ respectively to (17) and (18), together with the change of variables $K=F V, Q^{(l)}=V^{T} P^{(l)} V$ and $W^{(l)}(\cdot)=V^{T} T^{(l)}(\cdot) V$ for $V=G^{-1}$, leads to the following synthesis-oriented condition: a robust stabilization gain exists if there exist $V \in \mathbf{R}^{n \times n}, K \in \mathbf{R}^{m \times n}, Q^{(l)} \in \mathbf{S}_{+}^{n \times n}, W^{(l)}: \boldsymbol{\Xi} \rightarrow$ $\mathbf{S}^{n \times n}(l=1, \ldots, L)$, and $\lambda \in(0,1)$ satisfying

$$
\begin{aligned}
& E\left[W^{(l)}\left(\xi_{0}\right)\right] \leq 0 \quad(l=1, \ldots, L), \\
& {\left[\begin{array}{cc}
W^{(l)}\left(\xi_{\star}\right)+\lambda^{2} Q^{(l)} & 0 \\
0 & -Q^{(l)}
\end{array}\right]} \\
& +\operatorname{He}\left(\left[\begin{array}{c}
A_{\mathrm{s}}^{T} \\
I_{n}
\end{array}\right]\left[A_{\mathrm{op}}^{(l)}\left(\xi_{\star}\right) V+B_{\mathrm{op}}^{(l)}\left(\xi_{\star}\right) K V\right]\right) \geq 0 \\
& \left(l=1, \ldots, L ; \forall \xi_{\star} \in \boldsymbol{\Xi}\right) .
\end{aligned}
$$

A robust stabilization gain can be obtained from the solution of this inequality condition as $F=K V^{-1}$. If we take $A_{\mathrm{s}}=0$, the above condition immediately reduces to that in Theorem 3 in Hosoe et al. (2018). Hence, the above condition is a generalization of the earlier result. Such a new condition, however, is also incompatible with numerical synthesis as in the case of robust stability analysis based on Theorem 2. Hence, we further derive an equivalent numerically-tractable inequality condition from the above condition by using Lemmas 1 and 2 .

First, under the additional constraint

$$
V+V^{T}-Q^{(l)}>0(l=1, \ldots, L),
$$

the inequality condition (49) and (50) can be equivalently transformed to

$$
\begin{aligned}
& E\left[\lambda^{2} Q^{(l)}+\operatorname{He}\left(A_{\mathrm{s}}^{T}\left(A_{\mathrm{op}}^{(l)}\left(\xi_{\star}\right) V+B_{\mathrm{op}}^{(l)}\left(\xi_{\star}\right) K\right)\right)\right. \\
& -\left(V^{T} A_{\mathrm{s}}+A_{\mathrm{op}}^{(l)}\left(\xi_{\star}\right) V+B_{\mathrm{op}}^{(l)}\left(\xi_{\star}\right) K\right)^{T}\left(V+V^{T}-Q^{(l)}\right)^{-1} \\
& \left.\cdot\left(V^{T} A_{\mathrm{s}}+A_{\mathrm{op}}^{(l)}\left(\xi_{\star}\right) V+B_{\mathrm{op}}^{(l)}\left(\xi_{\star}\right) K\right)\right] \geq 0 \quad(l=1, \ldots, L)
\end{aligned}
$$

by using Lemma 1 and the Schur complement technique. We see that this inequality involves decision variables contained in the expectation operation. Hence, we next consider applying Lemma 2 to take out the decision variables from the expectation operation (recall the arguments in Subsection 3.2). Then, such transformation of the inequality and the application of the Schur complement technique finally lead us to the following theorem. 
Theorem 4 Suppose $\xi$ satisfies Assumption 1 and $A_{\mathrm{op}}^{(l)}\left(\xi_{k}\right)$ and $B_{\mathrm{op}}^{(l)}\left(\xi_{k}\right)(l=1, \ldots, L)$ satisfy Assumption 3. There exists a gain $F$ such that the closed-loop system (1)-(3) and (47) is exponentially stable in the second moment robustly with respect to $\mathbf{E}^{L}$ if there exist $V \in \mathbf{R}^{n \times n}, K \in \mathbf{R}^{m \times n}, Q^{(l)} \in \mathbf{S}_{+}^{n \times n}(l=1, \ldots, L)$ and $\lambda \in(0,1)$ satisfying (51) and

$$
\begin{aligned}
& {\left[\begin{array}{cc}
\lambda^{2} Q^{(l)} & 0 \\
0 & -Q^{(l)} \otimes I_{\bar{m}_{l}}
\end{array}\right]} \\
& +\operatorname{He}\left(\left[\begin{array}{c}
A_{\mathrm{s}}^{T} \otimes \bar{Y}_{1}^{(l) T} \\
I_{n \bar{m}_{l}}
\end{array}\right]\left[\widetilde{Y}_{A}^{(l)} V+\widetilde{Y}_{B}^{(l)} K V \otimes I_{\bar{m}_{l}}\right]\right) \\
& \geq 0(l=1, \ldots, L)
\end{aligned}
$$

for a given matrix $A_{\mathrm{s}} \in \mathbf{R}^{n \times n}$, where $\bar{Y}_{1}^{(l)}, \tilde{Y}_{A}^{(l)}$ and $\widetilde{Y}_{B}^{(l)}$ are given as

$\widetilde{Y}_{A}^{(l)}:=\left[\begin{array}{c}\bar{Y}_{A 1}^{(l)} \\ \vdots \\ \bar{Y}_{A n}^{(l)}\end{array}\right] \in \mathbf{R}^{n \bar{m}_{l} \times n}, \quad \widetilde{Y}_{B}^{(l)}:=\left[\begin{array}{c}\bar{Y}_{B 1}^{(l)} \\ \vdots \\ \bar{Y}_{B n}^{(l)}\end{array}\right] \in \mathbf{R}^{n \bar{m}_{l} \times m}$,

$\bar{Y}_{1 A B}^{(l)}=:\left[\bar{Y}_{1}^{(l)}, \bar{Y}_{A 1}^{(l)}, \ldots, \bar{Y}_{A n}^{(l)}, \bar{Y}_{B 1}^{(l)}, \ldots, \bar{Y}_{B n}^{(l)}\right]$

$\left(\bar{Y}_{1}^{(l)} \in \mathbf{R}^{\bar{m}_{l} \times 1}, \bar{Y}_{A i}^{(l)} \in \mathbf{R}^{\bar{m}_{l} \times n}, \bar{Y}_{B i}^{(l)} \in \mathbf{R}^{\bar{m}_{l} \times m}\right.$

$(i=1, \ldots, n))$

with full row rank matrices $\bar{Y}_{1 A B}^{(l)} \in \mathbf{R}^{\bar{m}_{l} \times\left(n^{2}+n m+1\right)}$ satisfying

$$
\begin{aligned}
\bar{Y}_{1 A B}^{(l) T} \bar{Y}_{1 A B}^{(l)}= & E\left[Y_{1 A B}^{2(l)}\left(\xi_{0}\right)\right], \\
Y_{1 A B}^{2(l)}\left(\xi_{0}\right):= & {\left[1, \operatorname{row}\left(A_{\mathrm{op}}^{(l)}\left(\xi_{0}\right)\right), \operatorname{row}\left(B_{\mathrm{op}}^{(l)}\left(\xi_{0}\right)\right)\right]^{T} } \\
& \cdot\left[1, \operatorname{row}\left(A_{\mathrm{op}}^{(l)}\left(\xi_{0}\right)\right), \operatorname{row}\left(B_{\mathrm{op}}^{(l)}\left(\xi_{0}\right)\right)\right] .
\end{aligned}
$$

In particular, $V$ becomes nonsingular, and $F=K V^{-1}$ is one such state feedback gain.

As in the case of Theorem 3, the inequality condition in the above theorem can be solved as a standard LMI (for each fixed $\lambda$ ) once $\bar{Y}_{1}^{(l)}, \widetilde{Y}_{A}^{(l)}$ and $\tilde{Y}_{B}^{(l)}(l=1, \ldots, L)$ are calculated. Hence, we can design a robustly stabilizing state feedback by using this theorem, without using the samples of $\xi_{0}$. The increase of conservativeness in this inequality condition, compared to that in Theorem 3 , could be led only by the fixed $A_{\mathrm{s}}$ in (48). In other words, if $A_{\mathrm{s}}$ is also regarded as a decision variable, the synthesisoriented inequality condition in Theorem 4 is theoretically no more conservative than the analysis-oriented inequality condition in Theorem 3 (although appropriate $A_{\mathrm{s}}$ is generally difficult to globally search for).

\section{Numerical Example}

This section demonstrates effectiveness of the robust stability analysis and synthesis framework developed with the linearization technique using two lemmas, through the comparison with the authors' earlier approach in Hosoe et al. (2018). Let us consider the process $\xi$ satisfying Assumption 1 given by

$$
\xi_{k}=\left[\xi_{1 k}, \xi_{2 k}\right]^{T}, \quad \xi_{1 k} \sim \mathrm{U}(1.1,1.4), \quad \xi_{2 k} \sim \mathrm{N}\left(0.6,0.3^{2}\right)
$$

(i.e., $Z=2$ ) and the vertices

$$
\begin{aligned}
& A_{\mathrm{op}}^{(l)}\left(\xi_{k}\right)=\left[\begin{array}{ccc}
0.6 & 0 & a_{2 k}^{(l)} \\
-0.4 & a_{1 k}^{(l)} & 0 \\
0 & -0.4-a_{1 k}^{(l)} a_{2 k}^{(l)}
\end{array}\right], \\
& B_{\mathrm{op}}^{(l)}\left(\xi_{k}\right)=[1,0,0]^{T}(l=1, \ldots, 4), \\
& a_{1 k}^{(1)}=a_{1 k}^{(3)}=\xi_{1 k}, \quad a_{1 k}^{(2)}=a_{1 k}^{(4)}=\xi_{1 k}-0.3, \\
& a_{2 k}^{(1)}=a_{2 k}^{(2)}=0.6, \quad a_{2 k}^{(3)}=a_{2 k}^{(4)}=\xi_{2 k}
\end{aligned}
$$

(i.e., $L=4$ ) for the open-loop system (3), (43)-(45). This system was dealt with in Hosoe et al. (2018) and its $A$ matrix $A_{\mathrm{op}}\left(\xi_{k} ; \theta\right)$ can be identified with the uncertain random matrix (5) under $\theta^{(1)}=\left(1-\delta_{1}\right)\left(1-\delta_{2}\right), \theta^{(2)}=$ $\delta_{1}\left(1-\delta_{2}\right), \theta^{(3)}=\left(1-\delta_{1}\right) \delta_{2}$ and $\theta^{(4)}=\delta_{1} \delta_{2}$.

We first briefly revisit the numerical results obtained in the earlier study (Hosoe et al., 2018) for the above system. In the earlier study, we carried out synthesis and post-synthesis analysis through approximating the distribution of $\xi_{0}$ with its samples (i.e., with the empirical distribution), although the details are omitted not to make the arguments verbose. Such a samplebased method then led us in the study to the gain $F=$ $[-1.4267,2.9341,0.0609]$ as the optimal solution (i.e., that minimizing $\lambda$ ) for the synthesis under 100 generated samples of $\xi_{0}$ (and some restriction on mappings corresponding to $W^{(l)}$ in (49) and (50), which was needed at the numerical stage in association with the calculation of the confidence level in the post-synthesis analysis). The computation time for this synthesis was 70.06 s under the environment with MATLAB, YALMIP (Löfberg, 2004) and SDPT3 (Tütüncü et al., 2003) running on a laptop equipped with 8.00 GB RAM and Intel(R) Core(TM) i7$5600 \mathrm{CPU} @ 2.60 \mathrm{GHz}$; we also used this environment for computations in this paper. Since the above synthesis result depended on the generated samples, postsynthesis analysis was additionally performed with 1000 different samples of $\xi_{0}$, and $\lambda_{\text {est }}=0.7492$ was obtained as an estimate of the true minimal $\lambda$ (denoted by $\lambda_{\min }$ ) satisfying the earlier robust stability condition (with a restriction on mappings); this estimate corresponds to that of an upper bound of minimal $\lambda$ satisfying (6) for all $\theta \in \mathbf{E}^{4}$. With that estimate and some other information, 
the confidence level for $\lambda_{\min }<1$ (i.e., robust stability) was calculated and confirmed to be sufficiently high.

For the same system, we next design a robustly stabilizing gain $F$ with Theorem 4 . Before solving the LMI, we need to calculate the LMI coefficients $\bar{Y}_{1}^{(l)}, \widetilde{Y}_{A}^{(l)}$ and $\widetilde{Y}_{B}^{(l)}(l=1, \ldots, L)$ given by $(54)-(57)$. This calculation can be numerically performed with MATLAB and Symbolic Math Toolbox, and we obtained all the coefficients in $38.38 \mathrm{~s}$ under the computation environment described above. The associated ranks $\bar{m}_{l}$ became 2 for $l=1$ and 2 , and 4 for $l=3$ and 4 , which are less than the worst case value $n^{2}+n m+1=13$ in the sense of the size of the expectation in (56) for the present situation. With such coefficients and $A_{\mathrm{s}}=0$, we minimized $\lambda$ under (51) and (53) through a bisection with respect to $\lambda$. Then, we obtained 0.6837 as the minimal $\lambda$, and the solution leading to the gain $F=K V^{-1}=[-1.0678,2.5312,1.6770]$. In the bisection for the synthesis, we solved the LMI seventeen times, and the average time of the single LMI computation was $0.3079 \mathrm{~s}$. Since the above value of minimal $\lambda$ is the result obtained under the fixed $A_{\mathrm{s}}$ (i.e., under the constraint on the $S$-variable), the post-synthesis analysis using Theorem 3 without such a constraint might provide us with a less conservative result. Hence, we numerically computed the minimization of $\lambda$ under (26) and (42) in a fashion similar to the above synthesis. Then, we obtained 0.6806 as the minimal $\lambda$, which is less than the value obtained at the synthesis stage.

As we can see, the new robust stability analysis and synthesis framework successfully provided a better result (i.e., smaller $\lambda$ ) than that in Hosoe et al. (2018) revisited above, despite no need to use the sample-based method; the value of minimal $\lambda$ obtained in the new framework is not an estimate, and readily corresponds to an upper bound of minimal $\lambda$ satisfying (6) for all $\theta \in \mathbf{E}^{4}$. Possible reasons for this improvement could be attributed to a quality issue of the generated samples of $\xi_{0}$ in the earlier study and the use of less conservative inequality conditions in the new framework; in the present situation, the latter reason is related essentially only with the restriction on mappings needed to introduce in the earlier study, since we took $A_{\mathrm{s}}=0$ above (recall the arguments below (50)). In summary, we can say that although the earlier framework has an advantage that the exact model of the distribution of $\xi_{0}$ is not necessarily required (since only samples of $\xi_{0}$ are used), the new framework provides us with more accurate and less conservative results when the LMI coefficients can be calculated.

Remark 3 In the synthesis using Theorem 4, we have a degree of freedom in the selection of $A_{\mathrm{s}}$. By exploiting this degree of freedom, we can actually further improve the results in the new framework. As we can see from (48), $S_{1}$ and $S_{2}$ in Theorem 3 correspond to $G A_{\mathrm{s}}^{T}$ and $G$, respectively. Hence, if we take $A_{\mathrm{s}}=\left(S_{2}^{-1} S_{1}\right)^{T}$ with $S_{1}$ and $S_{2}$ obtained in the post-synthesis analysis, and if we design the gain $F$ with such $A_{\mathrm{s}}$ again, we can guarantee that the obtained result at least does not become worse than that in the analysis (since a trivial solution always exists). By using this idea once in the above numerical example, we obtained $F=[-1.0494,2.5652,1.8049]$, which led us to 0.6794 as the minimal $\lambda$ under (26) and (42).

\section{Conclusion}

This paper proposed a technique of using two key lemmas for linearizing parameter-dependent inequality conditions with decision variables contained in the expectation operation for discrete-time linear systems represented with random polytopes. As a demonstration of usefulness of the technique, we derived with the lemmas new numerically-tractable LMI conditions for robust stability of the systems. The obtained LMI conditions correspond to a solid stochastic counterpart (in other words, a generalization in the stochastic sense) of one of the latest robust stability results for deterministic systems (Ebihara et al., 2015). Hence, they enable us to achieve the same level of robust stability analysis and synthesis as such latest deterministic systems results, despite our dealing with systems represented with random polytopes. The improvement of the inequality conditions from the viewpoint of conservativeness was numerically confirmed through comparison with our earlier result. The proposed linearization technique is expected to contribute to deriving LMI conditions not only for the present robust stability problem but also for other robust control problems, and would facilitate the future development of the LMI-based control framework for the systems represented with random polytopes.

\section{Acknowledgements}

This work was partially supported by JSPS KAKENHI Grant Number 17K14700.

\section{References}

Arnold, L. (1998). Random Dynamical Systems. Berlin Heidelberg, Germany: Springer-Verlag.

Boyd, S., El Ghaoui, L., Feron, E., \& Balakrishnan, V. (1994). Linear Matrix Inequalities in System and Control Theory. Philadelphia, PA, USA: SIAM.

Costa, O. L. V., \& Figueiredo, D. Z. (2014). Stochastic stability of jump discrete-time linear systems with Markov chain in a general Borel space. IEEE Transactions on Automatic Control, 59, 223-227.

Costa, O. L. V., Fragoso, M. D., \& Marques, R. P. (2005). Discrete-Time Markov Jump Linear Systems. London, UK: Springer-Verlag.

De Koning, W. L. (1992). Compensatability and optimal compensation of systems with white parameters. IEEE Transactions on Automatic Control, 37, 579588. 
Ebihara, Y., Peaucelle, D., \& Arzelier, D. (2015). SVariable Approach to LMI-Based Robust Control. London, UK: Springer-Verlag.

Evensen, G. (2003). The ensemble Kalman filter: Theoretical formulation and practical implementation. Ocean Dynamics, 53, 343-367.

Finkelstein, M. (2008). Failure Rate Modelling for Reliability and Risk. London, UK: Springer-Verlag.

Hosoe, Y., \& Hagiwara, T. (2019). Equivalent stability notions, Lyapunov inequality, and its application in discrete-time linear systems with stochastic dynamics determined by an i.i.d. process. IEEE Transactions on Automatic Control, 64, 4764-4771.

Hosoe, Y., Hagiwara, T., \& Peaucelle, D. (2018). Robust stability analysis and state feedback synthesis for discrete-time systems characterized by random polytopes. IEEE Transactions on Automatic Control, 63, $556-562$.

Hug, D. (2013). Random polytopes. In E. Spodarev (Ed.), Stochastic Geometry, Spatial Statistics and Random Fields: Asymptotic Methods (Lecture Notes in Mathematics) (pp. 205-238). Berlin Heidelberg, Germany: Springer-Verlag.

Klenke, A. (2014). Probability Theory: A Comprehensive Course. (2nd ed.). London, UK: Springer-Verlag.

Kozin, F. (1969). A survey of stability of stochastic systems. Automatica, 5, 95-112.

Löfberg, J. (2004). YALMIP: A toolbox for modeling and optimization in MATLAB. In Proc. 2004 IEEE International Symposium on Computer Aided Control Systems Design (pp. 284-289).

Paxson, V., \& Floyd, S. (1995). Wide area traffic: The failure of Poisson modeling. IEEE/ACM Transactions on Networking, 3, 226-244.

de Souza, C. E. (2006). Robust stability and stabilization of uncertain discrete-time Markovian jump linear systems. IEEE Transactions on Automatic Control, $51,836-841$.

Tütüncü, R. H., Toh, K. C., \& Todd, M. J. (2003). Solving semidefinite-quadratic-linear programs using SDPT3. Mathematical Programming Series B, 95, 189-217.

Van Willigenburg, L. G., \& De Koning, W. L. (1999). Optimal reduced-order compensation of time-varying discrete-time systems with deterministic and white parameters. Automatica, 35, 129-138. 